\title{
Phlyctenulosis-Like Presentation Secondary to Embedded Corneal Foreign Body
}

\author{
Valliammai Muthappan', Jared G. Smedley², Carlton R. Fenzl1, Majid Moshirfar ${ }^{3 *}$ \\ ${ }^{1}$ John A. Moran Eye Center, University of Utah, Salt Lake City, USA \\ ${ }^{2}$ College of Human Medicine, Michigan State University, Lansing, USA \\ ${ }^{3}$ Department of Ophthalmology, Francis I. Proctor Foundation, University of California San Francisco, \\ San Francisco, USA \\ Email: ${ }^{*}$ Majid.Moshirfar@ucsf.edu
}

Received 6 August 2014; revised 7 September 2014; accepted 3 October 2014

Copyright (C) 2014 by authors and Scientific Research Publishing Inc.

This work is licensed under the Creative Commons Attribution International License (CC BY). http://creativecommons.org/licenses/by/4.0/

(c) (i) Open Access

\begin{abstract}
Case Presentation: A nine-year-old boy presented to the general ophthalmologist with a several weeks history of redness, photophobia and intermittent foreign body sensation in the right eye. A pigmented lesion with anterior chamber inflammation was noted on examination. B-scan ultrasound was performed and revealed no foreign body. The patient was diagnosed with anterior uveitis, which did not completely respond to treatment. The differential diagnosis was expanded to include peripheral ulcerative keratitis, phlyctenulosis, pigmented neoplasm, and corneal foreign body. Upon referral to a cornea specialist, an exam under anesthesia revealed a large foreign body consistent with a rock fragment in the peripheral cornea, which was subsequently removed without complication. Conclusion: This case highlights an atypical presentation of foreign body as well as a differential diagnosis of pigmented peripheral corneal lesions. Foreign bodies represent the most common cause of urgent ophthalmic evaluation. When evaluating lesions of the cornea, it is imperative to keep an extensive differential diagnosis, giving the potential for severe and rapid development of visually threatening complications.
\end{abstract}

\section{Keywords}

Corneal Foreign Body, Phlyctenulosis, Pigmented Corneal Lesion, Anterior Uveitis

\section{Introduction}

Peripheral corneal inflammatory lesions are a group of conditions with similar signs and symptoms. Limbal and

\footnotetext{
${ }^{*}$ Corresponding author.
}

How to cite this paper: Muthappan, V., Smedley, J.G., Fenzl, C.R. and Moshirfar, M. (2014) Phlyctenulosis-Like Presentation Secondary to Embedded Corneal Foreign Body. Open Journal of Ophthalmology, 4, 112-116. 
perilimbal injection, corneal neovascularization, corneal infiltrate, and anterior chamber inflammation are all findings commonly seen. The identification of a correct diagnosis begins with a complete history and physical exam along with necessary medical and/or surgical therapies. We report a case of unilateral peripheral corneal foreign body accompanied by injection, corneal neovascularization, and intraocular inflammation.

\section{Case Presentation}

A nine-year-old boy presented to the general ophthalmologist with a several week history of redness, photophobia and occasional foreign body sensation in the right eye. The patient's history was significant for playing outside on a windy day a few days prior to symptom development. Upon evaluation, uncorrected visual acuity (UCVA) was 20/25 in the right eye and 20/20 in the left. Slit lamp examination revealed anterior chamber inflammation with a dark brown lesion in the superotemporal cornea of the right eye. B-scan ultrasound was performed and revealed no foreign body. The patient was diagnosed with acute anterior uveitis with peripheral anterior synechiae. He was treated with prednisolone acetate $1 \%$ ophthalmic drops four times a day; cyclopentolate $1 \%$ ophthalmic drops twice a day and erythromycin ointment.

One week later, the patient noticed some improvement of his symptoms. Visual acuity was unchanged and gonioscopy revealed a pigmented lesion within the temporal corneal stroma, not extending beyond the endothelium. The pigmented area was no longer considered to be a peripheral anterior synechiae, and the differential diagnosis was expanded to include peripheral ulcerative keratitis, phlyctenulosis, and pigmented neoplasm, in addition to corneal foreign body. The patient was referred to a corneal specialist for further evaluation and treatment.

The patient was seen in our clinic two days later. Right UCVA was 20/20-2 and left UCVA was 20/15-2. Slit lamp examination of the right cornea revealed a $1.4 \mathrm{~mm}$ by $2 \mathrm{~mm}$ lesion at the ten o'clock position just inside the limbus (Figure 1(a)). The lesion had surrounding neovascular pannus and lipid deposition (Figure 1(b)). The anterior chamber was deep and quiet, and the iris was round and reactive. Given the patient's age, wind exposure and incomplete response to treatment, a foreign body was suspected and evaluation under anesthesia (EUA) was scheduled.

Eight days later, the patient underwent EUA and a foreign body was identified in the peripheral cornea in the area of the pigmented lesion. The foreign body was estimated to extend $90 \%$ through the cornea. It was removed without complication and identified by pathology as a fragment of rock (Figure 2(a)). The patient was started

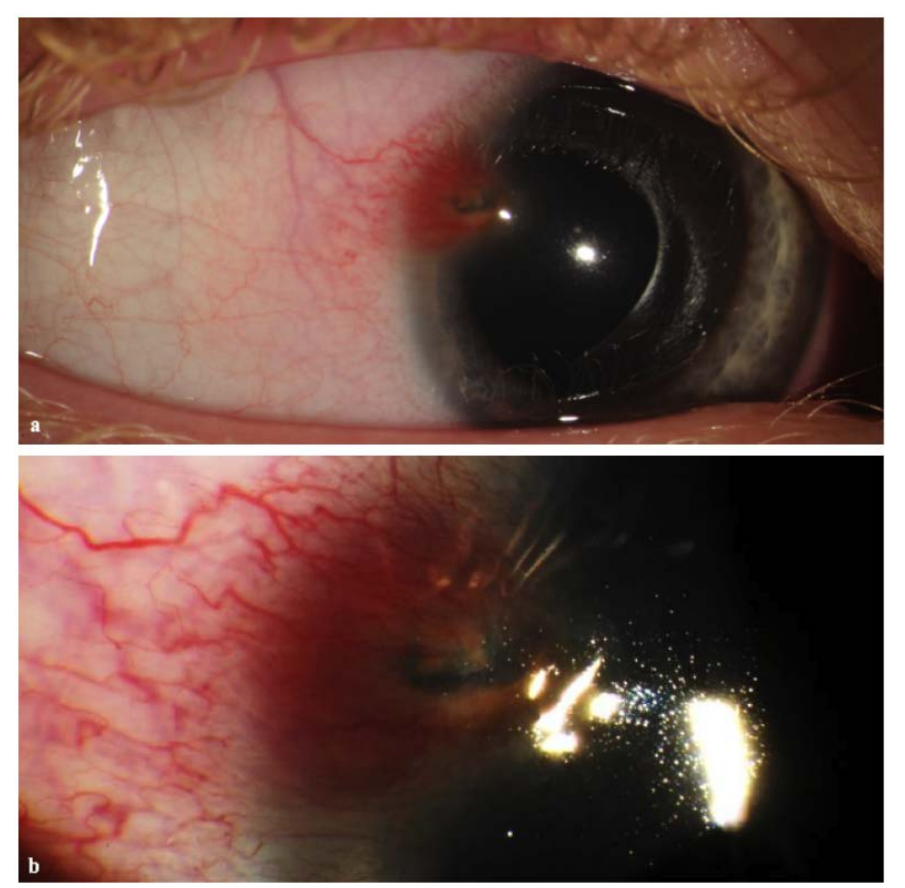

Figure 1. (a) Slit-lamp photograph showing corneal lesion with surrounding neovascular pannus and lipid deposition; (b) Higher magnification slit-lamp photograph of the right eye showing neovascularization and pannus formation. 

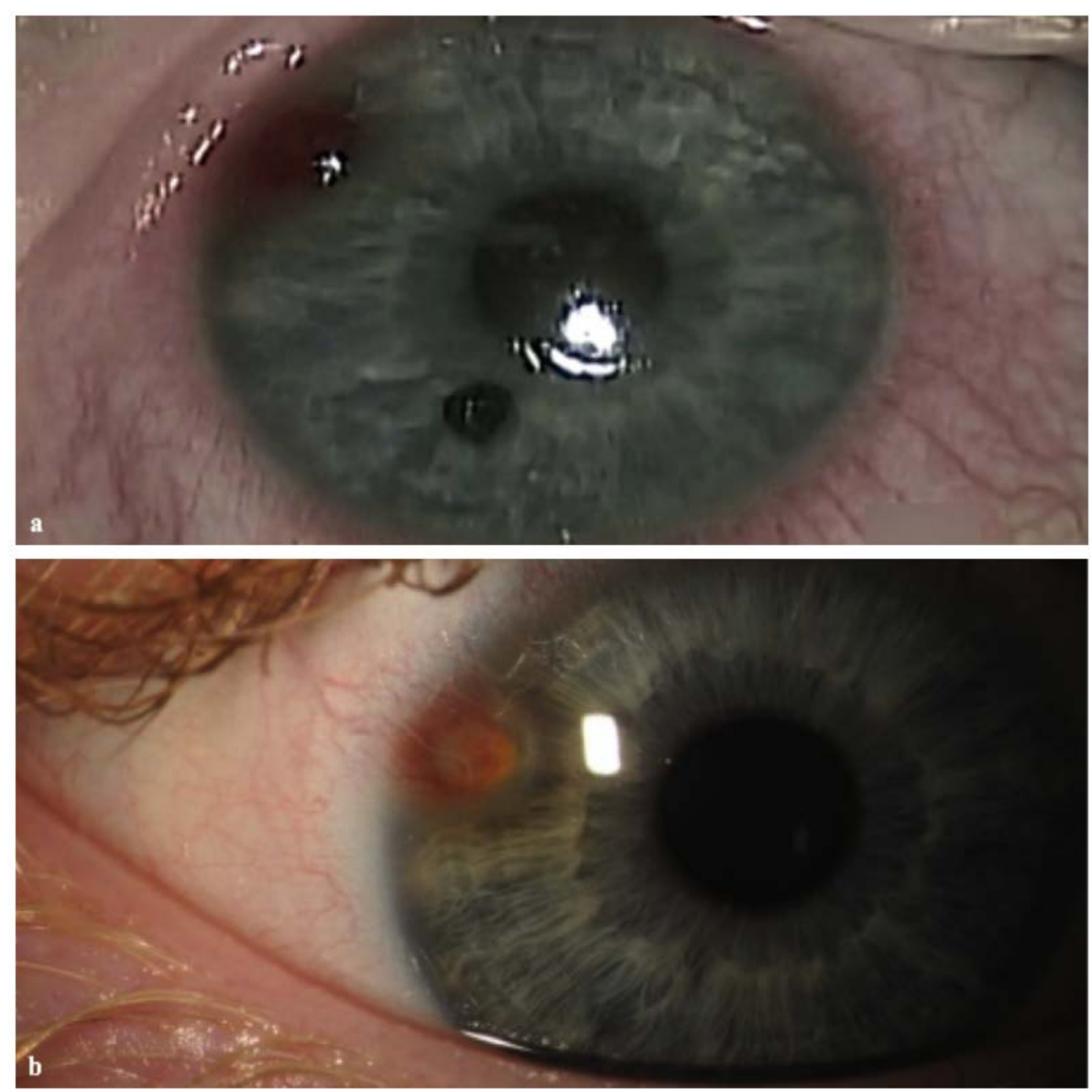

Figure 2. (a) Intraoperative photo of the corneal foreign body (yellow arrow) removed and placed on the inferior cornea at the seven o'clock position for size comparison. Surgical wound (white arrow) is visible in right superotemporal position; (b) One-week post-operative photo depicting a resolving lesion (white arrow) with a persistent peripheral lipid deposition.

on gatifloxacin $0.5 \%$ ophthalmic drops four times a day. Prednisolone acetate $1 \%$ was continued four times a day and cyclopentolate was discontinued. At one week post-operatively, the redness and irritation of the right eye had resolved and UCVA was 20/20. A healing corneal wound is visible in the superotemporal cornea (Figure 2(b)).

\section{Discussion}

We present a case of a pigmented peripheral corneal lesion associated with redness, irritation and foreign body sensation in a pediatric patient with history of sandstorm exposure. The diagnosis was confounded by the negative ultrasound. Ultrasound biomicroscopy has greater sensitivity than basic ultrasound for detecting ocular foreign bodies [1] [2]; however, this could not be performed due to patient's age and compliance. In this case, the diagnosis was not apparent until the fragment of rock was surgically identified and removed. This case illustrates an unusual presentation of a common ophthalmologic problem. This patient's corneal lesion on slit-lamp examination appeared similar to several other ocular disorders, with which the patient was diagnosed and briefly treated for, prior to the EUA.

Initially, this patient was diagnosed with peripheral anterior synechiae secondary to acute anterior iritis, which is somewhat uncommon in the pediatric population. Inflammatory causes of anterior uveitis include juvenile idiopathic arthritis (JIA), psoriatic arthritis, and tubulointerstitial nephritis and uveitis syndrome (TINU). Infectious causes such as herpetic uveitis and lyme disease are also important to consider [3]. Figure 3(b) shows a slit-lamp image of an unrelated patient with peripheral anterior synechiae secondary to inflammation, with iris 

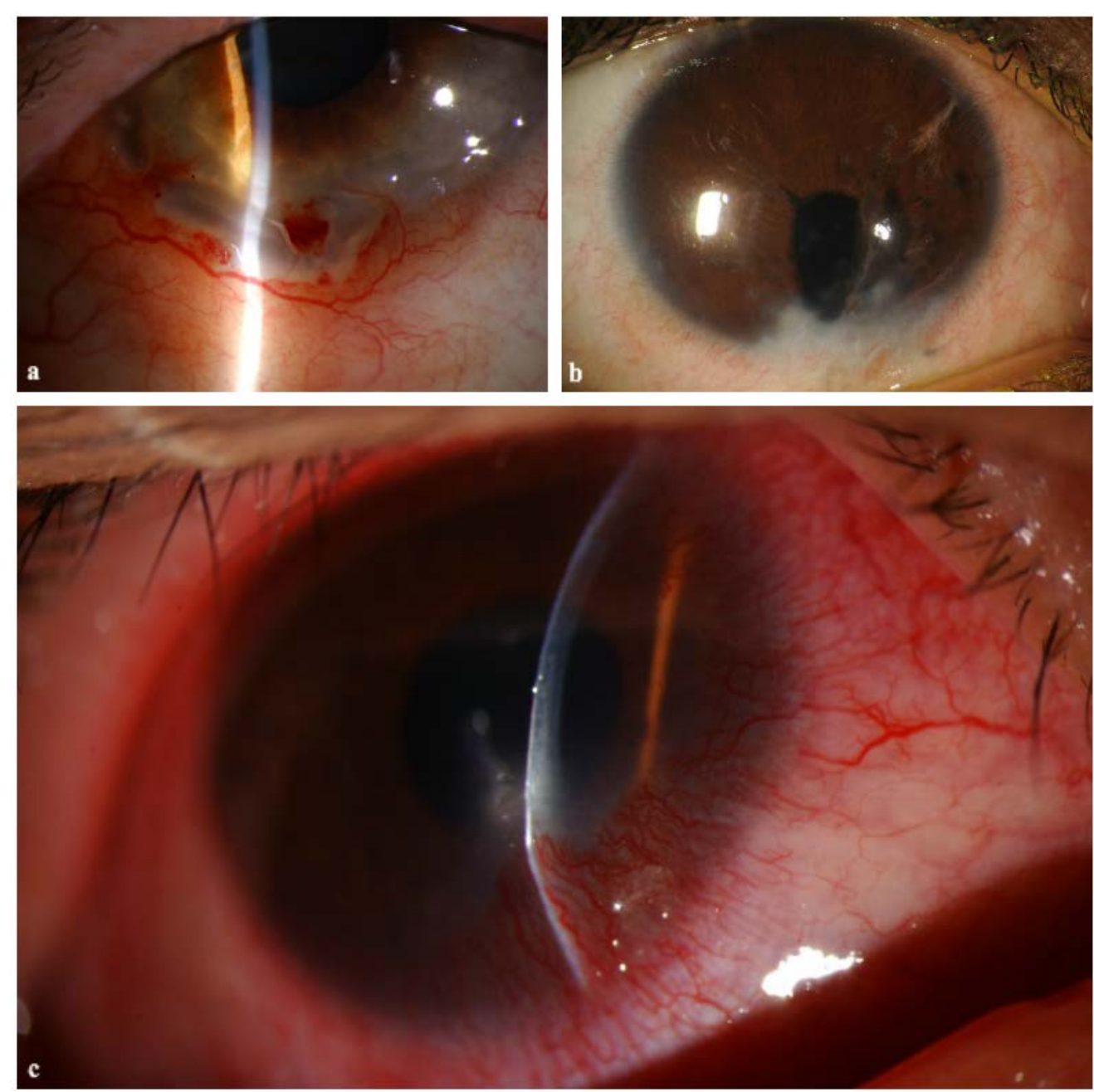

Figure 3. (a) Peripheral ulcerative keratitis. Ulcerations and stromal thinning are seen in the inferotemporal limbus. Conjunctival vasodilation and surrounding neovascularization are also seen; (b) Peripheral anterior synechiae with inflammation secondary to trauma. Irregularities of the iris are often seen with anterior synechiae; (c) Phlyctenulosis due to staphylococcus hypersensitivity. Wedge shaped neovascularization with pannus formation is visible on the inferonasal peripheral cornea. A white nodule is seen at leading edge of lesion centrally.

irregularities, somewhat resembling the peripheral corneal lesion in our patient (Figure 1(a)). Peripheral ulcerative keratitis (PUK) was also entertained as a diagnosis, due to the presence of a peripheral corneal lesion associated with redness, irritation and photophobia. It is extremely rare in children, but classically manifests as peripheral corneal infiltrates that lead to epithelial breakdown and frank ulceration [4] [5]. A slit-lamp image of a true peripheral ulcerative keratitis (Figure 3(a)) showing limbal ulceration, conjunctival injection and pannus, is shown for comparison to our patient's corneal foreign body slit-lamp image (Figure 1(a)). Both anterior uveitis and PUK were unlikely because of no previous history of systemic or infectious disease.

This patient was ultimately referred to us for a peripheral corneal lesion resembling phlyctenulosis. Phlyctenulosis is typically caused by a delayed (type IV) hypersensitivity reaction to staphylococcal blepharitis or tuberculosis (TB) [6]. Patients often have a history of chronic blepharitis and/or recurrent chalazia or TB exposure [7]. Corneal phlyctenules appear as white nodules at the limbus with surrounding dilated conjunctival blood vessels. Severe forms may present more centrally with accompanied corneal neovascularization. Figure 3(c) shows an example of a patient with phlyctenulosis to exemplify the similarities between this presentation and our patient's corneal lesion. This patient had no history of blepharitis or risk factors for TB exposure, which makes phlyctenulosis a less likely cause of the corneal lesion in our patient. 


\section{Conclusion}

In the end, the patient was found to have a corneal foreign body, likely acquired due to flying debris in the sandstorm. Corneal foreign body is consistent with this patient's history of foreign body sensation, redness and irritation, and the examination findings of a pigmented corneal lesion. It is also consistent with the patient demographics, as $47 \%$ of emergency eye consults in children involve injuries that increase the risk for foreign body [8]. The foreign body was removed and the patient has recovered nicely. This case provides an interesting differential diagnosis of similarly appearing peripheral corneal lesions in a pediatric patient.

\section{References}

[1] Wang, Z., Jiang, H., Kang, Y. and Chen, X. (1999) The Use of Ultrasound Biomicroscope in the Diagnosis of Anterior Segment Intraocular Foreign Bodies. Yan Ke Xue Bao, 15, 236-237.

[2] Kaushik, S., Ichhpujani, P., Ramasubramanian, A. and Pandav, S.S. (2008) Occult Intraocular Foreign Body: Ultrasound Biomicroscopy Holds the Key. International Ophthalmology, 28, 71-73. http://dx.doi.org/10.1007/s10792-007-9110-5

[3] American Academy of Ophthalmology (2011) Pediatric Ophthalmology and Strabismus. Basic and Clinical Science Course, Section 6. American Academy of Ophthalmology, 267-272.

[4] Leung, A.K., Mireskandari, K. and Ali, A. (2011) Peripheral Ulcerative Keratitis in a Child. Journal of AAPOS, 15, 486-488. http://dx.doi.org/10.1016/j.jaapos.2011.06.009

[5] Krachmer, J.H., Mannis, M.J. and Holland, E.J. (2011) Cornea: Fundamentals, Diagnosis and Management. 3rd Edition, Elsevier Inc., New York, 1121-1122.

[6] Gerstenblith, A. and Rabinowitz, M. (2012) Cornea: Phlyctenulosis. The Wills Eye Manuel: Office and Emergency Room Diagnosis and Treatment of Eye Disease. 6th Edition, Lippincott, Williams and Wilkins, Philadelphia, 17-19, 89-90.

[7] Zaidman, G.W. (2011) The Pediatric Corneal Infiltrate. Current Opinion in Ophthalmology, 22, 261-266. http://dx.doi.org/10.1097/ICU.0b013e3283479ffc

[8] Upshaw, J.E., Brenkert, T.E. and Losek, J.D. (2008) Ocular Foreign Bodies in Children. Pediatric Emergency Care, 24, 409-414. http://dx.doi.org/10.1097/PEC.0b013e318177a806 
Scientific Research Publishing (SCIRP) is one of the largest Open Access journal publishers. It is currently publishing more than 200 open access, online, peer-reviewed journals covering a wide range of academic disciplines. SCIRP serves the worldwide academic communities and contributes to the progress and application of science with its publication.

Other selected journals from SCIRP are listed as below. Submit your manuscript to us via either submit@scirp.org or Online Submission Portal.
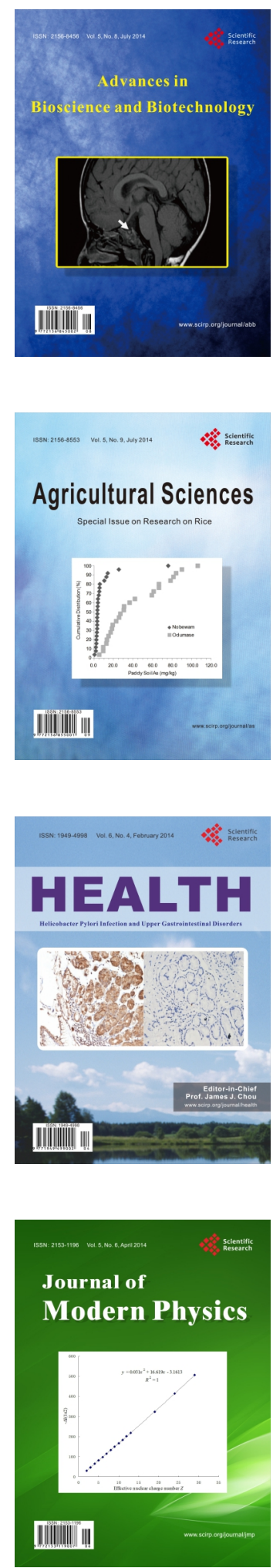
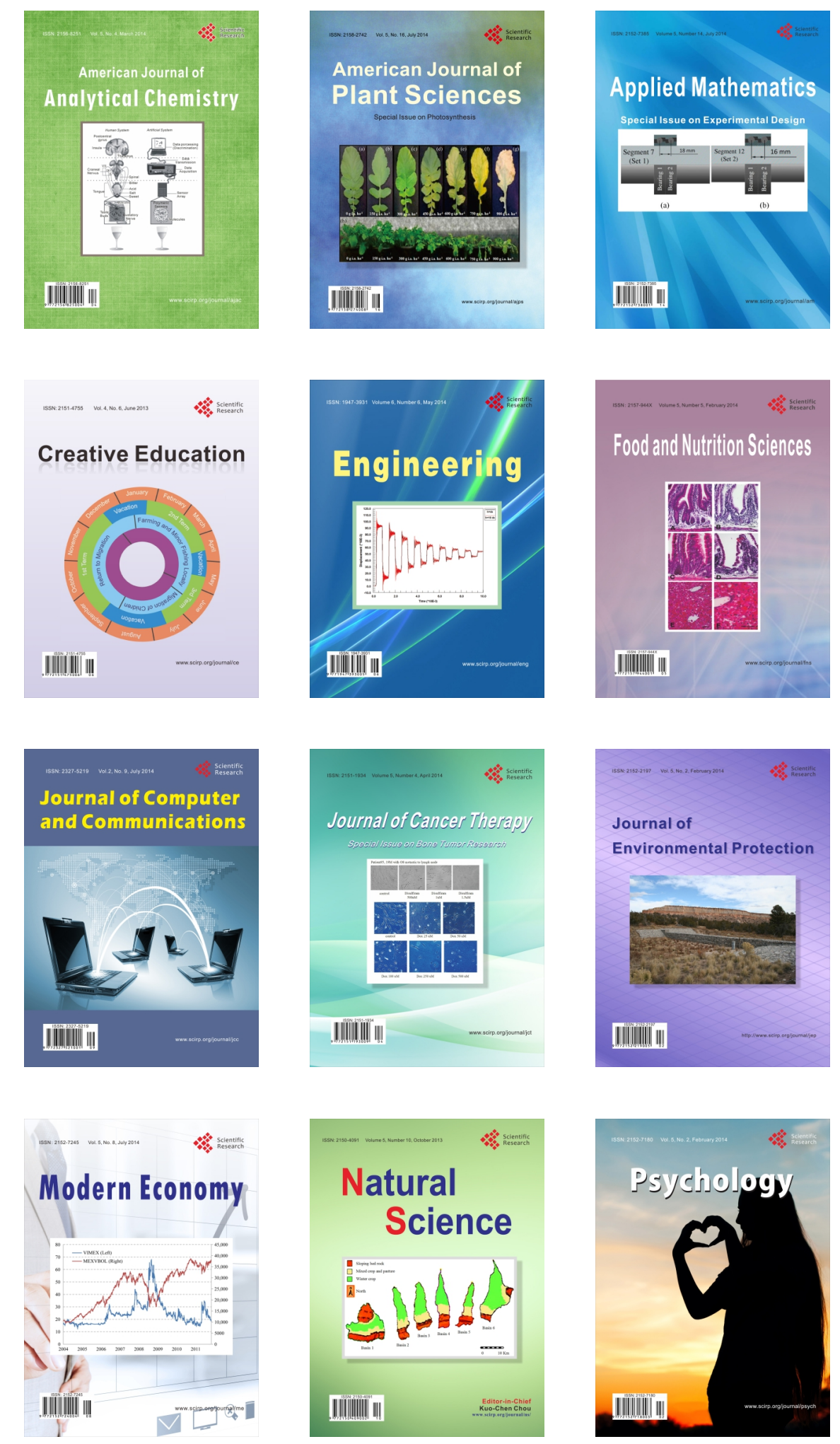